MS23-P02

\section{Towards automated data collection for continuous rotation electron diffraction: current status and development}

\author{
Bin Wang ${ }^{1}$, Stef Smeets ${ }^{1}$, Hongyi Xu ${ }^{1}$, Xiaodong Zou ${ }^{1}$ \\ 1. Department of Materials and Environmental Chemistry, \\ Stockholm University, Stockholm, Sweden \\ email: bin.wang@mmk.su.se
}

Electron diffraction tomography has been developing during the past 10 years and is proven to be very helpful in crystal structure determination [1], especially for beam sensitive materials such as zeolites, metal-organic frameworks (MOFs), and even proteins. The development of data collection methods went from discrete sampling of the reciprocal space (ADT, RED, etc.) to continuous data collection [2] with the help of fast electron counting detectors. Structure solution usually requires high tilt range and minimal dynamical effects from electrons, while for beam sensitive materials it is also desired to have minimum beam damage to the sample. The conventional discrete sampling methods suffer in this case from higher beam damage because of longer electron exposure time. The continuous method is thus preferable because it not only reduces data collection time, but also integrates the reflection intensities over the exposure time interval, which improves the accuracy of intensity extraction. However, the method is highly limited by the mechanical instabilities of goniometers inside the transmission electron microscopes (TEM) that the crystals can easily move out of the beam during data collection.

Here we illustrate the current status of the continuous rotation electron diffraction (cRED) method by showing that refinement against only electron diffraction data has been feasible. Datasets with high tilt ranges (i.e. $>100^{\circ}$ ) were collected for a MOF sample, Co-CAU-36, on different crystals and initial structure solution for the datasets from SIR2014 showed clearly the existence of the porphyrins and even guest molecules in the pores. By comparing refinement results from different individual datasets, the deviation for framework atoms was on average $0.04 \AA$, while for atoms in the guest molecules the deviation was on average $0.11 \AA$. Furthermore, the whole process from TEM sample preparation to the refined structure took only 9 hours.

However, collection of high quality cRED data still requires reasonable experience, i.e. blindly tracking back the crystal which might be moving out of the beam is not very straightforward. With development of electron diffraction data collection softwares such as Instamatic [3], automatic tracking back of the crystals becomes feasible by taking a defocused diffraction image regularly, performing cross correlation for how much the crystal has moved, and apply lens change to make the beam chase the crystal. In the cost of losing around $10 \%$ of diffraction data, higher tilt range can be achieved in a fully automated manner. This allows a normal electron diffraction experimentalist to collect datasets with higher tilt ranges. Further development is still needed and we aim to build up a fully automatic cRED data collection protocol, with automated crystal finding and continuous rotation data collection.
References:

[1] Yun, Y. et al. (2015). IUCrJ.2, 267-282.

[2] Gemmi, M. et al. (2015). J. Appl. Cryst. 48, 718-727.

[3] Smeets, S. et al. (2017). Instamatic (Version 0.4). Zenedo. http:// doi.org/10.5281/zenodo.1090389.

Keywords: Continuous rotation electron diffraction, method development, automation 